\title{
Energy spectra of quantum rings
}

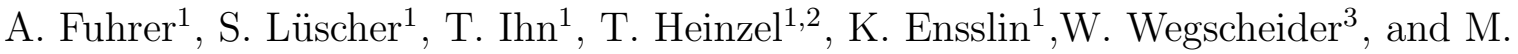 \\ Bichler $^{4}$ \\ 1 Solid State Physics Laboratory, ETH Zürich, 8093 Zürich, Switzerland, \\ 2 Angewandte und Experimentelle Physik, Universität Regensburg, 93040 Regensburg, Germany, \\ 3 Walter Schottky Institut, TU München, 85748 Garching, Germany, \\ ${ }^{4}$ Fakultät für Physik, Universität Freiburg i. Br., 79104 Freiburg, Germany
}

(October 22, 2018)

\begin{abstract}
Ring geometries have fascinated experimental and theoretical physicists over many years. Open rings connected to leads allow the observation of the Aharonov-Bohm effect [1], a paradigm of quantum mechanical phase coherence [2,3]. The phase coherence of transport through a quantum dot embedded in one arm of an open ring has been demonstrated [4. The energy spectrum of closed rings [5] has only recently been analysed by optical experiments [6,7] and is the basis for the prediction of persistent currents [8] and related experiments [9 11]. Here we report magnetotransport experiments on a ring-shaped semiconductor quantum dot in the Coulomb blockade regime [12]. The measurements allow us to extract the discrete energy levels of a realistic ring, which are found to agree well with theoretical expectations. Such an agreement, so far only found for few-electron quantum dots, is here extended to a many-electron system [13]. In a semiclassical language our results indicate that electron motion is governed by regular rather than chaotic motion, an unexplored regime in many-electron quantum dots.
\end{abstract}


Quantum ring samples have been fabricated on AlGaAs-GaAs heterostructures containing a two-dimensional electron gas (2DEG) with density $5 \times 10^{15} \mathrm{~m}^{-2}$ and mobility $900^{\prime} 000 \mathrm{~cm}^{2} / \mathrm{Vs}$ at $\mathrm{T}=4.2 \mathrm{~K}$ only $34 \mathrm{~nm}$ below the sample surface. The surface of the heterostructure has been locally oxidized by applying a voltage between the conductive tip of an atomic force microscope (AFM) and the 2DEG [14]. The electron gas is depleted below the oxidized regions, which was used in other studies for defining high quality quantum dots [15]. The details of the fabrication process which is crucial for the high-electronic quality of the quantum ring, are described in Ref. [16]. Figure 1(a) shows an AFM image (taken with an unbiased tip directly after the oxidation process) of the oxide lines defining the quantum ring. The width of the quantum point contacts connecting the ring to source (drain) is controlled by voltages applied to the lateral gate electrodes qpc1a and b (qpc2a and b). The number of electrons in the ring can be tuned via the lateral plunger gates pg1 and 2. Shape deformations due to applied in-plane gate voltages are known to be relatively weak [15,16]. The schematic in Fig. 1(b) shows the dimensions of the quantum ring. After the oxidation step the sample has been covered with a metallic top gate electrode. With the combination of in-plane and top gate electrodes the quantum ring can be tuned into the Coulomb blockade regime with the single-particle level spacing being much larger than the thermal energy $\mathrm{kT}$. Figure 2(b) presents a colour plot of the current through the quantum ring as a function of plunger-gate voltage and magnetic field $\mathrm{B}$ (applied normal to the 2DEG plane). This measurement was performed at a source-drain voltage $\mathrm{V}_{\mathrm{SD}}=20 \mu \mathrm{V}$ and at a temperature of $100 \mathrm{mK}$ in a dilution refrigerator. In Fig. 2(a) the Coulomb blockade oscillations have been extracted along the horizontal dashed line in Fig. 2(b), i.e. at constant B=92 mT. From corresponding measurements of the Coulomb blockade diamonds we determine a charging energy $\mathrm{E}_{\mathrm{C}}=\mathrm{e}^{2} / \mathrm{C}_{\Sigma} \approx 190 \mu \mathrm{eV}$. The observed discrete level spacings after subtraction of Ec within the constant interaction model [12] can be as large as $\Delta \approx 180 \mu \mathrm{eV}$ (see below). From a simple capacitance model and the ring geometry we estimate that about 200 electrons are distributed on 2-3 radial subbands. In Fig. 2(b) the position as well as the amplitude of the Coulomb blockade peaks oscillate as a function magnetic field with a period of $\Delta \mathrm{B}=75 \mathrm{mT}$ (horizontal white lines) which is exactly the Aharonov-Bohm period for this ring. In fact, by opening the point contacts (not shown) we find the well-known Aharonov-Bohm oscillations in the conductance with the same $\Delta \mathrm{B}[2,3,17,18]$. The oscillations are visualized along a line of constant gate voltage in Fig. 2(c) (Fig. 2(b), vertical dashed line).

What determines the magnetic field dependence of positions and amplitudes of the Coulomb blockade resonances? We start the discussion from the energy spectrum of an infinitely thin perfect ring of radius $r_{0}$ enclosing $m$ flux quanta: [8]

$$
E_{m, l}=\frac{\hbar^{2}}{2 m^{\star} r_{0}^{2}}(m+l)^{2}
$$

Here $m^{\star}$ is the mass of the particle, $l$ is the angular momentum quantum number. For a given angular momentum state the energies as a function of magnetic field (or flux $m$ ) lie on a parabola with its apex at $m=-l$, as depicted in Figure 3(a). According to this simple picture a single Coulomb blockade peak should oscillate as a function of B along a zig-zag line (blue curve) with the Aharonov-Bohm period $\Delta \mathrm{B}$. Comparison with the measurement in Fig. 2(b) shows that indeed some peaks move along a zig-zag line, but others show barely any B-dependence, a behaviour which will be discussed later. 
We first take a closer look at the h/e-periodic modulation of the Coulomb blockade peak amplitude. A peak follows a line of constant electron number (blue line in Fig. 2(a)). The current is successively carried by states $(l),(-l-1),(l-1),(-l-2),(l-2)$, when B is increased from zero, i.e. a change in state occurs every half flux quantum. The amplitude of Coulomb blockade peaks is determined by the wave function overlap between the confined states in the ring and the extended states in source and drain [12]. However, the wave functions in an infinitely thin perfect ring are independent of magnetic field and given by

$$
\Psi_{l}(\Phi)=\frac{1}{\sqrt{2 \pi}} e^{i l \Phi}
$$

where $\Phi$ is the azimutal coordinate in the ring. The (lateral) overlap (proportional to $|\Psi|^{2}$ ) with source and drain is the same for all states. This model does therefore not predict the observed h/e-periodic modulation of the peak amplitude.

A more realistic but still analytically soluble model takes the finite extent of the wave functions in radial direction into account [19], which leads to multiple radial channels indexed by the quantum number n. Using this model and analysing the Coulomb diamonds in detail we obtain agreement with the previous estimate of about 2-3 radial modes and about 200 electrons in the ring. For small (large) n, the occupied states at the Fermi level have a larger (smaller) angular momentum quantum number $l$ at $\mathrm{B}=0$ and consequently display a stronger (weaker) magnetic field dispersion. This model predicts that the exponential decay of the wave functions in radial direction depends on the value of $l$ but is relatively insensitive to the value of $m$ (for small $m$ ). Since all states move in zig-zag lines with an $\mathrm{h} / \mathrm{e}$ periodicity in magnetic field, crossings of states with different angular momenta $l$ may lead to a different wave function overlap and therefore to a modulation of the corresponding Coulomb peak amplitude.

The angular uniformity of the probability density in a perfect ring stems from the cylindrical symmetry which, for the real sample, will be broken by the pure presence of source and drain, by dopants and by the limits of the fabrication procedure. This leads to pinning of the wave function and therefore to a distinct amplitude of the probability density at source and drain. The perturbation will become especially important at the degeneracy points of levels where it leads to anti-crossing behaviour [20]. In the simplest case the probability density changes from a uniform to a sinusoidal angle-dependence at the degeneracy points. In this picture the AB-periodic oscillation of the amplitude along a single Coulomb peak can be understood in terms of changing contributions of single particle levels to the current carrying state.

We now turn to the analysis of the experimental Coulomb blockade peak positions. They are obtained from measurements like the ones shown in Fig. 2(b) by converting the gatevoltage axis into an energy scale using the appropriate lever arm as determined from the analysis of the Coulomb blockade diamonds [21]. A constant charging energy of $190 \mu \mathrm{eV}$ is subtracted from the position of a Coulomb maximum [12,15 and the resulting energies are plotted in Fig. 3(c) as a function of magnetic field. Clearly many of the peaks move in pairs (see e.g. the black-purple, the blue-red or the green-purple pair), previously identified as spin pairs [15]. The exchange related spin splitting energy with a value of $\approx 20 \mu \mathrm{eV}$ for these peaks is on average smaller than the discrete energy level spacing $\Delta$. Electrons therefore successively populate these orbital states with spin-up and spin-down electrons. For other 
peaks (green, yellow, red) spin pairing is not clearly observed. As depicted in Fig. 3(c) the orbital states move up and down in magnetic field with the Aharonov-Bohm period $\Delta \mathrm{B}$. In this respect our experiments show the long-predicted energy spectrum characteristic for quantum rings [5].

Let us look at the states in Fig. 3(c) that have a very small dispersion in the magnetic field. In the framework of the model of Ref. [19] such 'flat' states can occur at the onset of the occupation of the next higher radial channel. However, in the experiment we observe such states over wide ranges of gate voltages and an extended model is necessary. In Fig. 3(b) we show a calculation with ring-parameters typical for our dot. The spectrum is obtained from the diagonalisation of a truncated Hamiltonian matrix expressed in the eigenstate-basis of Ref. [19], but including diagonal and off-diagonal elements given by a symmetry breaking potential [22]. Two radial modes are taken into account leading to two sets of parabolic energy dispersions as a function of magnetic field in the unperturbed system. The perturbation mixes states of positive and negative angular momenta, which leads in some cases to eigenenergies that barely depend on magnetic field. Such states can intersect the diamonds formed by strongly oscillating levels in close resemblance to the experimental findings. The model obviously can only give the general tendency of the experimental spectra. Nevertheless, the dominant deviations from the perfect ring spectrum can be understood as the result of a symmetry breaking potential perturbation.

We estimate the contribution of a particular strongly oscillating state to the persistent current [8] from the experimental dispersion (i.e. black dots in Fig. 3(c)) and obtain a value of $5 \mathrm{nA}$. If we assume that currents of all the lower lying states sum up to zero this current is also an estimate of the total persistent current in the ring and the value is consistent with previous magnetisation measurements [9 11].

For quantum dots containing a small number of electrons, the shell structure of the level occupancy can clearly be detected because of the dominating cylindrical symmetry [13]. Quantum dots containing many electrons are usually described in the context of an underlying classically chaotic geometry, since small perturbations of parameter space such as potential shape or magnetic field can induce parametric fluctuations in the energy levels and consequently in the Coulomb peak positions. Several theoretical and experimental publications address the question whether the spectra of such many-electron quantum dots can be adequately described by Random Matrix Theory [24. Our quantum ring represents a many-electron Coulomb blockaded system with regular geometry. This together with the small number of radial modes is the reason why we can qualitatively understand the principal features of the observed energy spectrum without the need of a statistical analysis. The source of this striking observation lies in the circular geometry of our ring.

In order to support this view we have fabricated a square shaped quantum dot with a circular antidot in the centre. This system is considered a Sinai billiard and is one of the theoretically best studied examples of a classically chaotic system. Fig. 4 shows the evolution of the conductance as a function of plunger gate and magnetic field of this system presented in a way comparable to Fig. 2(b) for the quantum ring. Around $\mathrm{B}=0$ the Coulomb peak maxima fluctuate irregularly as a function of magnetic field with an average period compatible with an Aharonov-Bohm-type argument. As the magnetic field is increased to a value, where the classical cyclotron diameter matches the antidots circumference, a well pronounced B-periodic behaviour of the Coulomb peak maxima in amplitude and position is 
recovered. Similar as in antidot lattices [25] the magnetic field is expected to induce regular parts in the predominately chaotic phase space existing at $\mathrm{B}=0$.

The detailed analysis of quantum rings demonstrates that even in many-electron Coulomb blockaded systems a detailed understanding of the energy spectrum can be obtained. With advanced fabrication techniques at hand this opens the path to the understanding of more complex and multiply connected structures on a quantum mechanical level. Electron-electron interactions beyond the constant interaction model are believed to play a minor role in our quantum ring. One indication is the observation of spin pairs and relatively small spin splitting. Once ring structures with only one radial mode occupied are available such quantum rings could be used to investigate spin effects [26] or even Luttinger liquid behaviour in a circular 1D system with periodic boundary conditions. One can also envision searching for persistent current effects in the transport signatures of a quantum ring now that the energy spectrum is experimentally accessible.

We thank M. Büttiker and D. Loss for valuable discussions. Financial support from the Swiss Science Foundation (Schweizerischer Nationalfonds) is gratefully acknowledged. 


\section{REFERENCES}

[1] Aharonov, Y., Bohm, D. Significance of Electromagnetic Potentials in the Quantum Theory. Phys. Rev. 115, 485-491 (1959).

[2] Webb, R. A., Washburn, S., Umbach, C. P., Laibowitz, R. B. Observation of h/e Aharonov-Bohm oscillations in normal-metal rings. Phys. Rev. Lett. 54, 2696-2699 (1985).

[3] Timp, G. et al. Observation of the Aharonov-Bohm effect for $\omega_{\mathrm{C}} \tau \geq 1$. Phys. Rev. Lett. 58, 2814-2817 (1987).

[4] Schuster, R. et al. Phase measurement in a quantum dot via a double-slit interference experiment. Nature 385, 417-420 (1997).

[5] Byers, N., Yang, C.N. Theoretical Considerations Concerning Quantized Magnetic Flux in Superconducting Cylinders. Phys. Rev. Lett. 7, 46-49 (1961).

[6] Lorke, A. et al. Spectroscopy of nanoscopic semiconductor rings. Phys. Rev. Lett. 84, 2223-2226 (2000).

[7] Warburton, R. J. et al. Optical emission from a charge-tunable quantum ring. Nature 405, 926 - 929 (2000).

[8] Büttiker, M., Imry, Y., Landauer, R. Josephson behavior in small normal onedimensional rings. Phys. Lett. 96A, 365-367 (1983).

[9] Lévy, L. P., Dolan, G., Dunsmuir, J., Bouchiat, H. Magnetization of mesoscopic copper rings: Evidence for persistent currents. Phys. Rev. Lett. 64, 2074-2077 (1990).

[10] Chandrasekhar, V. et al. Magnetic response of a single, isolated gold loop. Phys. Rev. Lett. 67, 3578-3581 (1991).

[11] Mailly, D., Chapelier, C., Benoit, A. Experimental observation of persistent currents in GaAs-AlGaAs single loop. Phys. Rev. Lett. 70, 2020-2023 (1993).

[12] Kouwenhoven, L.P. et al. Electron Transport in Quantum Dots. Nato ASI conference proceedings, ed. By L. P. Kouwenhoven, G. Schön, L.L. Sohn (Kluwer, Dordrecht, 1997), pp. 105-214.

[13] Tarucha, S., Austing, D.G., Honda, T., van der Haage, R.J., Kouwenhoven, L.P. Shell Filling and Spin Effects in a Few Electron Quantum Dot. Phys. Rev. Lett. 77, 36133616, (1996).

[14] Held, R. et al. In-plane gates and nanostructures fabricated by direct oxidation of semiconductor heterostructures with an atomic force microscope. Appl. Phys. Lett. 73, 262264 (1998).

[15] Lüscher, S., Heinzel, T., Ensslin, K., Wegscheider, W., Bichler M. Signatures of spin pairing in a quantum dot in the Coulomb blockade regime. Phys. Rev. Lett. 86, 2118$2121(2001)$.

[16] Heinzel, T. et al. Electronic Properties of Semiconductor Nanostructures patterned by AFM Lithography. Physica E 9, 84-93 (2001).

[17] Pedersen, S., Hansen, A. E., Kristensen, A., Sorensen, S. B., Lindelof, P. E. Observation of quantum asymmetry in an Aharonov-Bohm ring. Phys. Rev. B 61, 5457-5460 (2000).

[18] Cassé, M. et al. Temperature dependence of the Aharonov-Bohm oscillations and the energy spectrum in a single-mode ballistic ring. Phys. Rev. B 62, 2624-2629 (2000).

[19] Tan, W-C, Inkson, J. C. Electron states in a two-dimensional ring - an exactly soluble model. Semicon. Sci. Technol. 11, 1635-1641 (1996). 
[20] Chakraborty, T., Pietiläinen, P. Persistent currents in a quantum ring: Effects of impurities and interactions. Phys. Rev. B 52, 1932-1935 (1995).

[21] Kastner, M.A. The single-electron transistor. Rev. Mod. Phys. 64, 849-858 (1992).

[22] The angle independent part $\omega_{0}$ of Ref. [19 is replaced by $\omega_{0}(1+\epsilon \cos 2 \phi)$ following the idea of shape deformation in Ref. [23]. This breaks the symmetry leading to inter- as well as intra-subband coupling. The values used for the calculation were $\omega_{0}=2.73 \times 10^{12} \mathrm{~s}^{-1}$, $\mathrm{r}_{0}=132 \mathrm{~nm}$ and $\eta=0.06$.

[23] Berman, D., Entin-Wohlman, O., Azbel, M.Y. Diamagnetic spectrum and oscillations in an elliptic shell. Phys. Rev. B 42, 9299-9306 (1990).

[24] Beenakker, C. W. J. Random-matrix theory of quantum transport. Rev. Mod. Phys. 69, 731-808 (1997).

[25] Schuster, R., Ensslin, K. Antidot superlattices: classical chaos and quantum transport. Advances in Solid State Physics (Festkörperprobleme) 34, 195-218 (1994).

[26] Loss, D., Goldbart, P. Period and amplitude halving in mesoscopic rings with spin. Phys. Rev. B 43, 13762-13765 (1991). 


\section{FIGURES}

a

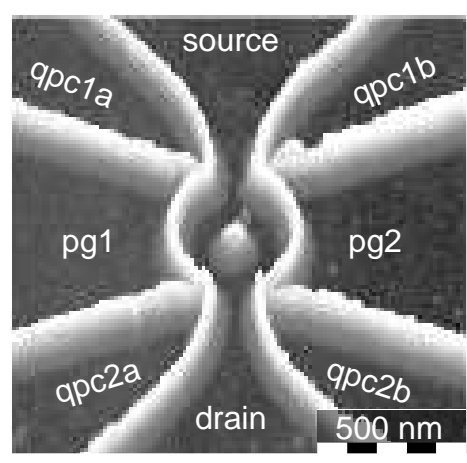

b

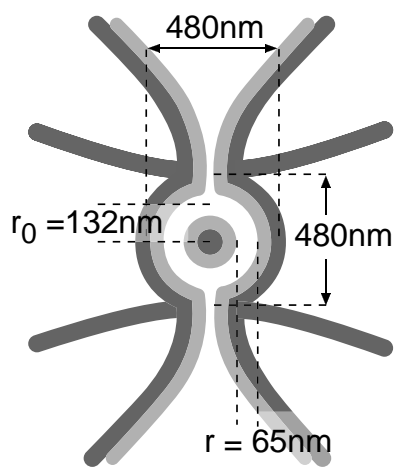

FIG. 1. Sample layout. a, Micrograph of the quantum ring taken with the unbiased AFM-tip after writing the structure. The oxide lines (bright regions) deplete the 2DEG 34nm below the surface separating the sample into several conductive (dark) regions. The current is passed from source to drain. The in-plane gates (qpc1a, qpc1b, qpc2a, qpc2b, pg1 and pg2) are used to tune the point contacts and two arms of the ring. b, Schematic sketch of the ring. The dark curves represent the oxide lines. From transmission measurements of the point contacts at source and drain we estimate the depletion length to be about $50 \mathrm{~nm}$ which results in an estimated channel width of $\Delta r \approx 65 \mathrm{~nm}$. The average radius of the ring is $\mathrm{r}_{0}=132 \mathrm{~nm}$. 


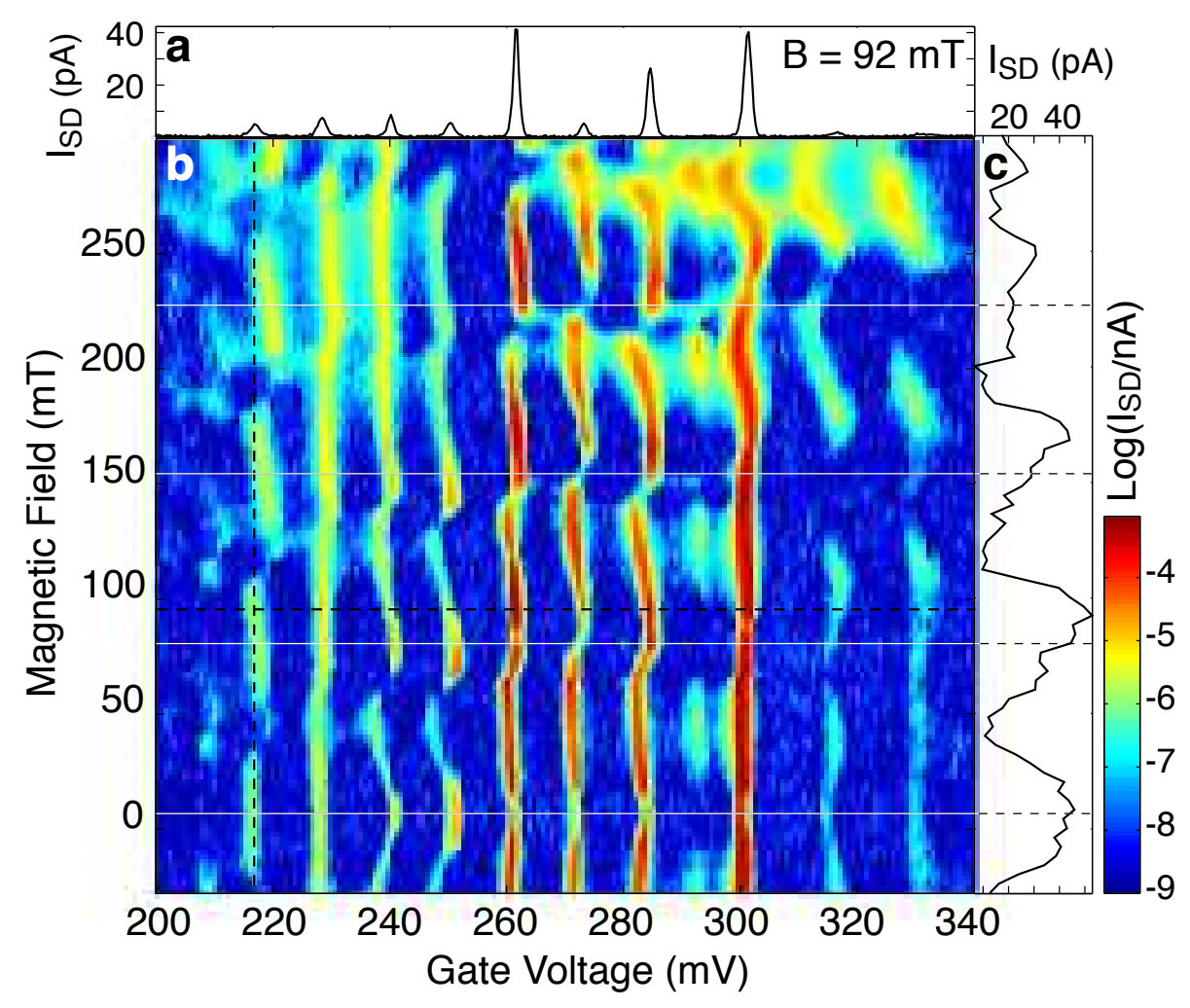

FIG. 2. The addition spectrum. a, Measurement of Coulomb blockade resonances at fixed magnetic field. The current is measured as a function of a voltage applied to both plunger gates (pg1 and 2) simultaneously. $\mathbf{b}$, The evolution of such sweeps with magnetic field results in the addition spectrum shown in colour. The regions of high current (yellow/red) mark configurations in which a bound state in the ring aligns with the Fermi level in source and drain. The Aharonov-Bohm period expected from the ring geometry is indicated by the thin white horizontal lines. c, Magnetic field sweep for constant gate voltage $\mathrm{Vpg}=218 \mathrm{mV}$ (dashed line in the colour plot). While this peak shows a maximum in amplitude for $\mathrm{B}=0$ other peaks $(\mathrm{Vpg}=270 \mathrm{mV})$ display a minimum in amplitude. 
a

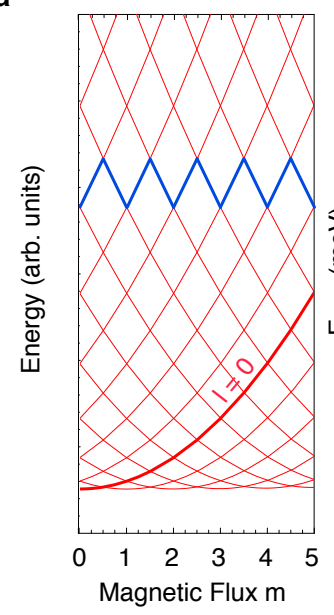

\section{b}

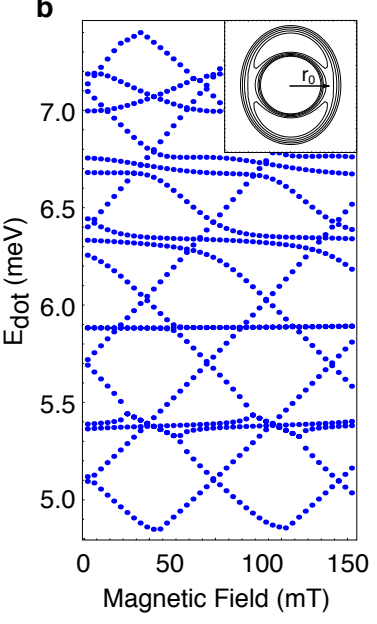

C

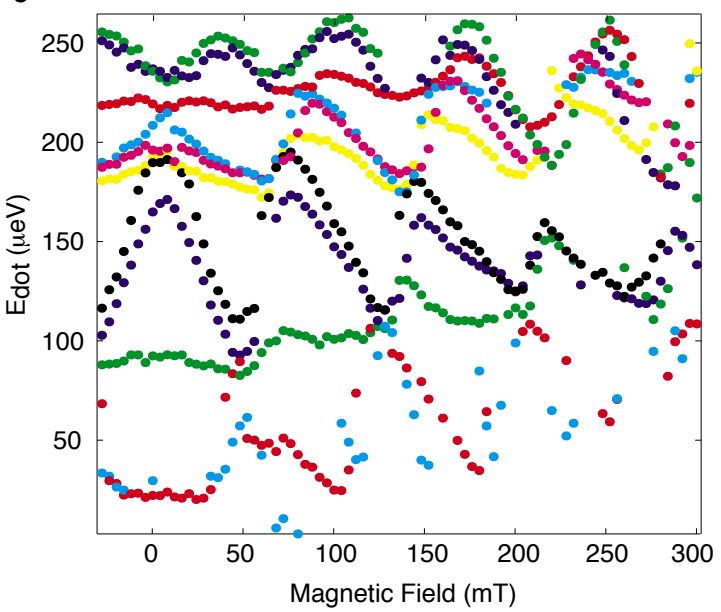

FIG. 3. Reconstruction of the energy spectrum. a, Theoretical spectrum of a single mode ring. The parabolas with constant $l$ (bold red line) have a minimum at $l=m$. The blue zig-zag line corresponds to a Coulomb peak after the charging energy has been subtracted in the constant interaction model. b, Calculated spectrum with ring parameters typical for our dot. We assume a slightly asymetric potential shown in the inset, which mixes states of positive and negative angular momenta. This leads in some cases to eigenenergies that barely depend on magnetic field. c, Reconstruction of the energy spectrum of the ring from the data shown in Figure 2. The plunger gate voltage was converted into dot energy using measurements of the Coulomb diamonds and a constant charging energy of $190 \mu \mathrm{eV}$ was subtracted. 


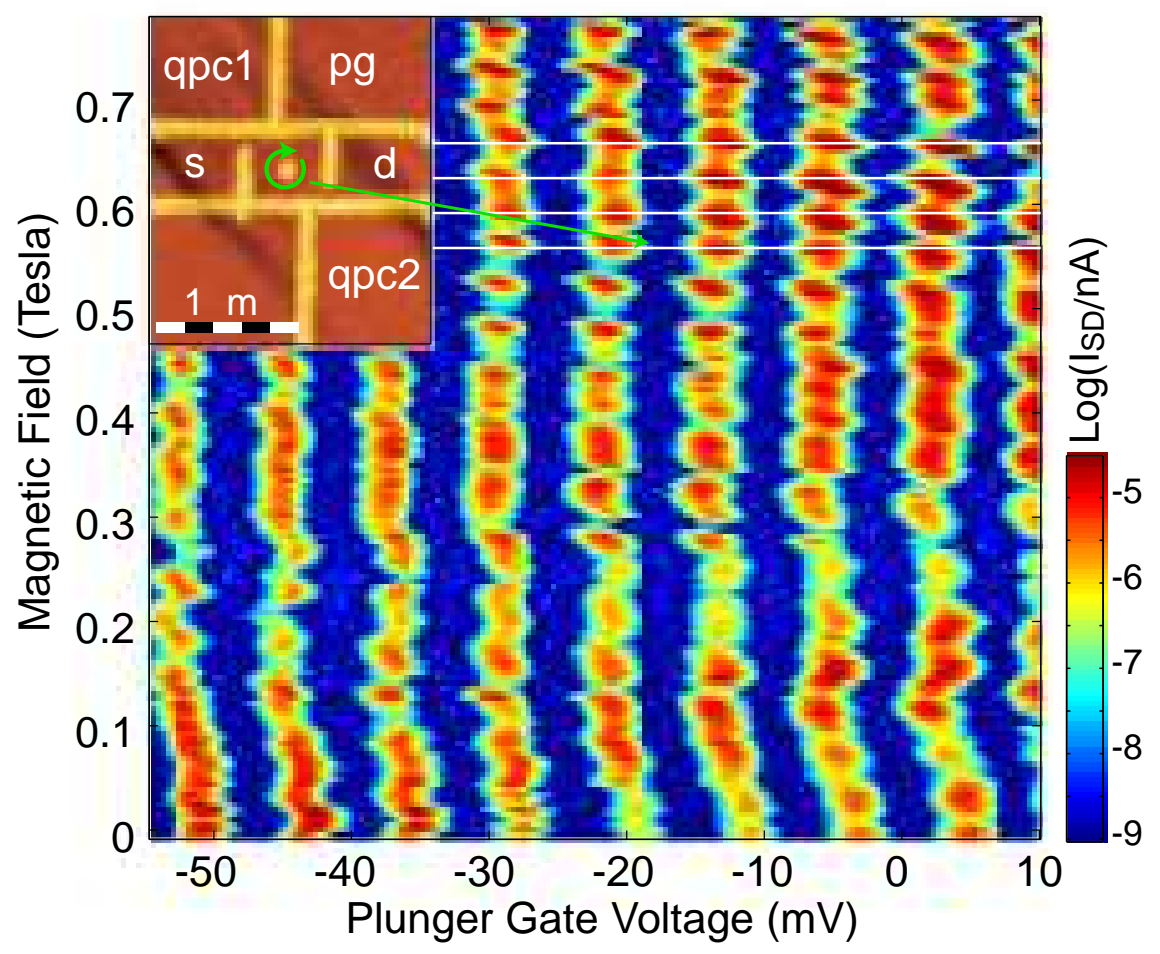

FIG. 4. Addition spectrum of a Sinai billiard. The inset shows an AFM-micrograph of the square shaped quantum dot with a circular antidot in the centre. The colour plot is a similar measurement to the one in Figure 2(b) and shows the evolution of the conductance as a function of plunger gate voltage and magnetic field. Around $\mathrm{B}=0$ the Coulomb maxima fluctuate irregularly as the magnetic field is changed. As the magnetic field is increased to a value, where the classical cyclotron orbit matches the antidots circumference (indicated by the green circle and the arrow in the inset), a well pronounced h/e-periodic behaviour (white lines) in amplitude and position of the Coulomb peaks is recovered, indicating quenching of the chaotic behaviour. 